\title{
Zu den Autor_innen
}

Carol Boyce Davies, PhD in Afrikanischer Literatur an der University of Ibadan (Nigeria), seit 2007 Professorin für Africana Studies und Englische Literatur an der Cornell University in Ithaka, New York. Zuvor baute sie an der Florida International University das African Diaspora Studies Programme auf und hatte diverse Gastprofessuren inne, z.B. an der Universität von Brazilia/Brasilien, der University of the West Indies in St. Augustine und der Peking Foreign Studies University in China. Ihre Forschungsschwerpunkte sind Afrikanische Diaspora, Schwarze Frauen in der transnationalen Politik, Literatur von schwarzen Frauen sowie Geschlechter-, Klassenund Macht-Verhältnisse in den USA und in der Karibik.

- ceb278@cornell.edu

Sabine Cassel-Bähr, Dipl.-Psych., Psychoanalytikerin DPV / IPV. Lehrtherapeutin und Supervisorin der Deutschen Gesellschaft für Sexualforschung (DGfS). Arbeitet in eigener Praxis in Hamburg. Themenschwerpunkte: (Weibliche) Sexualität und Perversion, Geschlechterverhältnis und Reproduktion sowie Ethik in der Psychoanalyse.

-scb@scasselbaehr.de

Nikita Dhawan, Dr. phil., Professorin für Politikwissenschaft und Gender Studies an der Justus-Liebig-Universität Gießen. Ihre Forschungs- und Interessenschwerpunkte liegen in den $\mathrm{Be}-$ reichen des Transnationalen Feminismus, der Globalen Gerechtigkeit, der
Menschenrechte sowie der Demokratie und Dekolonisierung. Sie erhielt 2017 den Käthe Leichter-Preis für ihre Forschungen im Bereich Frauen- und Geschlechterforschung sowie für die Förderung der Frauenbewegung und die Verdienste um die Geschlechtergleichstellung.

-dhawan.nikita@gmail.com

Esther M. Franke, M.A., Politik- und Kulturwissenschaftlerin, PhD Studentin am Department of Politics, New School for Social Research, New York. Arbeitsschwerpunkte: Feministische politische Theorie, transnationale Feminismen, Borders and Migration, Queer Theory.

- franke423@newschool.edu

Johanna Grubner, M.A., Universitätsassistentin am Institut für Soziologie der Johannes-Kepler-Universität Linz. Arbeitsschwerpunkte: Kritische Gesellschaftstheorie, Feministische Theorien und Geschlechterforschung, Hochschulforschung.

-johanna.grubner@jku.at

Margret Hauch, Dipl.-Psych., Psychologische Psychotherapeutin (TP), Sexualwissenschaftlerin, Lehrtherapeutin und Supervisorin der Deutschen Gesellschaft für Sexualforschung (DGfS), langjährige Mitarbeiterin am Institut für Sexualforschung und forensische Psychiatrie am Universitätsklinikum Eppendorf, z.Zt. arbeitet sie in freier Praxis Themenschwerpunkte: Heterosexualität, sexuelle Gewalt, Geschlechterver- 
hältnis, sogenannte sexuelle Lustlosigkeit und Paartherapie bei sexuellen Störungen.

-margret.hauch@hamburg.de

Ina Kerner, Dr. phil., Professorin für Dynamiken der Globalisierung und Leiterin des Seminars Politische Wissenschaft des Instituts für Kulturwissenschaft an der Universität KoblenzLandau, Campus Koblenz. Zuvor lehrte sie an den drei Berliner Universitäten, an der New School for Social Research in New York sowie an der Quaid-i-Azam University in Islamabad. Ihre Forschungsschwerpunkte liegen an der Schnittstelle von politischer Theorie, feministischer Theorie und postkolonialen Studien.

- kerner@uni-koblenz.de

Johanna Leinius, Dr. phil., M. Soc.Sci., wissenschaftliche Mitarbeiterin im Projekt "Ökologien des sozialen Zusammenhalts« am Fachbereich Gesellschaftswissenschaften der Universität Kassel. Arbeitsschwerpunkte: postkolonial-feministische Theorie, Bewegungsforschung, (Neo-) extraktivismus und Geschlecht.

-leinius@uni-kassel.de

Katharina Liebsch, Dr. phil. habil., arbeitet als Professorin für Soziologie unter besonderer Berücksichtigung der Mikrosoziologie an der Helmut Schmidt Universität/Universität der Bundeswehr Hamburg. Arbeitsschwerpunkte: Praxistheoretische Erforschung von Generationen- und Familienverhältnissen, geschlechtertheoretische Implikationen von Körper- und Biopolitik sowie die Analyse korrespondierender Wissensformen und Normen. -k.liebsch@hsu-hh.de
Christine Löw, Dr. phil., Politikwissenschaftlerin, zurzeit Gastprofessorin für "Geschlechterforschung und postkoloniale Theorie « am Institut für Politikwissenschaft der Eberhard-Karls-Universität Tübingen. Ihr derzeitiges Forschungsprojekt trägt den Titel "NaturGeschlecht-Gesellschaftliche Ungleichheiten". Arbeitsschwerpunkte: Feministische Theorien, Post-/Dekoloniale Ansätze, Entwicklungs- und Globalisierungsforschung, Klima-, Umweltund Ressourcenpolitik, Internationale Politische Ökonomie, New Materialism sowie Kritische Gesellschaftstheorie.

- christine.loew@uni-tuebingen.de

Rirhandu Mageza-Barthel, Dr. phil., ist Gastprofessorin für Internationale $\mathrm{Ge}-$ schlechterpolitik an der Universität Kassel. Ihre Forschung legt unter Bezugnahme auf transnationale Theorien und postkoloniale Ansätze einen Schwerpunkt auf normative Dynamiken in der internationalen Politik sowie in der Aushandlung und Umsetzung feministischer Politiken.

- mageza-barthel@uni-kassel.de

Amina Mama, Ph D, seit 2009 Professorin für Gender, Sexuality, and Women's Studies an der University of California, studierte Psychologie, Sozialpsychologie und Politische Wissenschaft. Sie promovierte am Birkbeck College der University of London mit der Arbeit "Race and Subjectivity: A Study of Black Women". Sie arbeitete als Wissenschaftlerin in Nigeria, Südafrika, Großbritannien, den Niederlanden und den USA. Im Sommer 2018 war sie Inhaberin der Angela Davis 
Gastprofessur am Cornelia Goethe Zentrum der Frankfurter Universität.

- amama@ucdavis.edu

Regine Othmer, Dipl.-Päd., Sozialwissenschaftlerin, freie Lektorin, Redakteurin und Übersetzerin, Herausgeberin der feministischen studien seit 1985.

- regine.othmer@gmail.com

Uta Ruppert, Prof. Dr. rer pol., Professorin für Politikwissenschaft und Politische Soziologie mit dem Schwerpunkt Globaler Süden und Gender und eine der Direktorinnen des Cornelia Goethe Centrums für Geschlechterforschung an der Goethe Universität Frankfurt/Main. Ihre Arbeitsschwerpunkte sind transnationale Feminismen, transnationale Solidaritäten, FrauenMenschenrechte und neuere SüdSüd Beziehungen.

-ruppert@soz.uni-frankfurt.de

Hanna Völkle, M. A. Politische Ökonomie, arbeitet als Politik- und Sozialwissenschaftlerin bei der EAF Berlin an den Schnittstellen von Vielfalt und Organisationsentwicklung. Sie hat einen Lehrauftrag an der Hochschule für Wirtschaft und Recht Berlin zu »Feminist and Ecological Economics».

- voelkle@eaf-berlin.de

Veronika Prieler, MSSc, Mitarbeiterin am Institut für Soziologie der JohannesKepler-Universität Linz, Arbeitsschwerpunkte: feministische Gesellschaftstheorie, Wohlfahrtsstaatsforschung, Care - veronika.prieler@jku.at

Tanja Scheiterbauer, Dr. phil., Post-Doc an der Goethe-Universität in Frankfurt a.M. am Schwerpunkt Entwick- lungsländer- und Geschlechterforschung. Sie forscht zu den Themen Geschlecht und Politik sowie soziale Bewegungen und Islamismus in Nordafrika und im Nahen Osten. Zurzeit befasst sie sich mit sozial-ökologischen Transformationsprozessen und Klimawandel in der Region.

- t.scheiterbauer@soz.uni-frankfurt.de

Eva Senghaas-Knobloch, Prof. (em.) Dr. phil., Universität Bremen, Forschungszentrum Nachhaltigkeit (artec), derzeitige Schwerpunkte: internationale und transnationale Regulierungen von Arbeit, fürsorgliche Praxis und nachhaltige gesellschaftliche Entwicklung. - esk@uni-bremen.de

Denise Siemer, Masterstudentin der Friedens- und Konfliktforschung an der Goethe Universität Frankfurt und studentische Mitarbeiterin an der Professur Globaler Süden und Gender im Fachbereich Gesellschaftswissenschaften. Ihre Forschungsinteressen liegen in der Wissenschaftstheorie, den black feminist theories, der Kritischen Theorie und den transnationalen feministischen Solidaritäten.

-denise-siemer@outlook.de

Christa Wichterich, Dr. phil., Soziologin, Scholar Activist, Publizistin, 2013/ 14 Gastprofessorin für Geschlechterpolitik an der Uni Kassel, früher Universitätsdozentin in Indien und im Iran, Auslandskorrespondentin für Ostafrika in Kenia, Teilnahme an Weltfrauen- und anderen internationalen Konferenzen.

-wichterich@femme-global.de 